\title{
IMAGING EVALUATION OF CARCINOMA STOMACH BY ULTRASONOGRAPHY AND COMPUTED TOMOGRAPHY- A COMPARATIVE STUDY OF 50 CASES
}

\author{
Mohsina Islam Bora1, Priyanka Priyadarshini Baishya²
}

${ }^{1}$ Registrar, Department of Radiodiagnosis, Gauhati Medical College and Hospital, Guwahati, Assam, India.

${ }^{2}$ Assistant Professor, Department of Radiodiagnosis, Gauhati Medical College and Hospital, Guwahati, Assam, India.

\section{BACKGROUND}

\section{ABSTRACT}

Stomach cancer is the second most common cancer among men and third most among women in Asia and worldwide. Almost all gastric cancers are adenocarcinomas (95\%). Other histological types include lymphomas and carcinoid tumours. The data obtained from the National Cancer Registries indicates that gastric cancer is a leading problem in North-Eastern and Southern states of Indian subcontinent.

The objective of our study was to evaluate the USG and CT imaging features of cases of stomach carcinoma referred to the Radiology Department, GMCH.

\section{MATERIALS AND METHODS}

This descriptive study of Ultrasonography and Computed Tomography were done in all patients of the study group. Histopathological study was done either by ultrasound-guided fine needle aspiration cytology, upper GI endoscopic biopsy or from post-operative specimen. Only histologically proven stomach carcinoma cases were included in our study. The age group of the patients ranged from 30 years to 90 years.

\section{RESULTS}

50 cases of stomach carcinoma were studied and among 50 cases 33 were male and 17 were female. Parameters like pattern of tumour growth, adjacent organ involvement, nodal spread and histological type of tumour were evaluated and tabulated.

\section{CONCLUSION}

Various findings like male predominance with male-to-female ratio of $1.9: 1,5^{\text {th }}-7^{\text {th }}$ decade as the age group with maximum number of cases etc. can be obtained from the present study. Computed Tomography is more accurate than ultrasonography in detecting extent of tumour, patterns of tumour growth, adjacent organ involvement, lymph node spread and metastasis.

\section{KEY WORDS}

Stomach Carcinoma, Ultrasonography, Computed Tomography.

HOW TO CITE THIS ARTICLE: Bora MI, Baishya PP. Imaging evaluation of carcinoma stomach by ultrasonography and computed tomography- a comparative study of 50 cases. J. Evolution Med. Dent. Sci. 2018;7(44):4785-4788, DOI: $10.14260 /$ jemds/2018/1067

\section{BACKGROUND}

Stomach cancer is the second most common cancer among men and third most among women in Asia and worldwide. The data obtained from the National Cancer Registries indicates that gastric cancer is a leading problem in NorthEastern and Southern states of Indian subcontinent. Gastric cancer is relatively rare before the age of 40 , but its incidence steadily climbs after that and peaks in the seventh decade of life. It is more common in men than women. Predisposing conditions that can increase the risk of developing gastric carcinoma include dietary factors, Helicobacter pylori gastritis, atrophic gastritis, pernicious anaemia, adenomatous gastric polyps and hereditary factors. Patients may present with dyspepsia, anorexia and weight loss as well as abdominal pain that is vague and insidious in nature. Nausea, vomiting and early satiety may occur with bulky tumours

'Financial or Other Competing Interest': None.

Submission 12-09-2018, Peer Review 11-10-2018,

Acceptance 17-10-2018, Published 29-10-2018.

Corresponding Author:

Priyanka Priyadarshini Baishya,

House No. 53, Tokobari Satra,

A. T. Road, Near Hotel Vishwaratna

Guwahati-781001, Assam, India.

E-mail: priyankapriyadarshini98@gmail.com

DOI: $10.14260 /$ jemds $/ 2018 / 1067$

\section{(c) $(1)$}

that obstruct the gastrointestinal lumen or infiltrative lesions that impair stomach distension.(1)

$50 \%$ of patients with stomach cancer are asymptomatic in the early stages and present as advanced gastric carcinoma (Invading into muscularis propria or beyond), which carries a much worse prognosis with a 5 years survival rate at about $60 \%$ or less.(2)

Adenocarcinoma is the most common histological type in 95\% of cases. However, other histological types include leiomyosarcoma, GI stromal tumour, lymphomas, secondaries and carcinoid tumours. In addition, there are a number of non-neoplastic tumour like lesions that should be considered in the differential diagnosis of stomach carcinoma like hyperplastic and hamartomatous polyp, inflammatory pseudotumour and ectopic pancreatic rest.

About $30 \%$ of gastric cancers are located in the antrum, $30 \%$ in the body and $30 \%$ in the fundus or cardia region. The remaining $10 \%$ are diffusely infiltrating lesions that involve the entire stomach. ${ }^{(3)}$

Multidetector Computed Tomography (MDCT) has a primary role in diagnosing and characterising gastric cancer as it offers an excellent evaluation of the gastric lumen, gastric wall and the adjacent structures. It also complements upper gastrointestinal endoscopy in establishing the diagnosis and defining the extent of the disease. 
Ultrasonography being readily available can be used for initial diagnosis in cases with clinical suspicion.

\section{Aims and Objectives}

1. To diagnose and characterise gastric carcinoma by ultrasonography and computed tomography.

2. To correlate ultrasonography and computed tomographic findings.

\section{MATERIALS AND METHODS}

This descriptive study was conducted in the Department of Radiology, Gauhati Medical College and Hospital from June 2016 to July 2017. 50 patients who were referred to the Department of Radiology from both indoor and outdoor units presenting with symptoms suspicious of stomach carcinoma and subsequently confirmed by HPE were included.

The age group of the patients ranged from 30 yrs. to 90 yrs. Both male and female sexes were studied.

\section{Methods}

Brief clinical history of the patients referred to the Department of Radiology with symptoms suggestive of or already diagnosed case of stomach carcinoma was taken. The history comprised of chief complaints, mode of onset, duration of illness, occupational history, personal history and past history. Both general, systemic and laboratory examinations of the patients were done.

\section{Imaging}

The patients were kept on empty stomach for at least 6 hours before the ultrasonographic examination. Real time B-mode ultrasonography was done with Antares Colour Doppler Ultrasound System (Make: Siemens) real time scanner and Samsung RS80A Ultrasound System.

For computed tomographic examinations, patients were again kept on empty stomach for at least $6 \mathrm{hrs}$. Written consent of the patient and attendants were taken for contrast examination. Before performing the scan, the procedure and objective were explained to the patient. Patients were given $750 \mathrm{~mL}$ of water, approximately 15 mins before scanning. An additional $250 \mathrm{~mL}$ along with effervescent was given immediately prior to the study to improve gastric distension. The machine used is Philips MX16 (16-Slice CT) scanner.

\section{CT Scan was performed using the Following Protocols-}

1. Positioning: Patients were scanned in the supine position with their arms above their heads. Scans were performed in the axial axis from cephalic to caudal levels. Prone imaging for better delineation of the proximal stomach was also used in selected cases.

2. Scanning: After positioning the patient, the topogram was taken. Spiral scanning was done. The abdomen scans were obtained as contiguous $5 \mathrm{~mm}$ slice. Both plain and contrast enhanced CT scans were obtained. $100 \mathrm{~mL}$ of a non-ionic water-soluble contrast medium were injected intravenously as a bolus dose to image the abdomen. Delayed sections through the abdomen were obtained to see the delayed enhancement pattern of the mass and excretion of both kidneys.

\section{Histopathological Examination}

These were performed either by USG guided or endoscopic fine needle aspiration cytology/ biopsy or from postoperative specimen.

\section{RESULTS}

Sex Distribution- In our present series 50 patients with stomach cancer were evaluated, out of which 33 were male and 17 were female. Males accounted for $66 \%$ and females accounted for $34 \%$ of the total number of cases.

Age Distribution- The age distribution was as per table below.

\begin{tabular}{|c|c|c|}
\hline Age Groups & No. of Cases & Percentage \\
\hline $30-40$ & 5 & 10 \\
\hline $41-50$ & 7 & 14 \\
\hline $51-60$ & 11 & 22 \\
\hline $61-70$ & 14 & 28 \\
\hline $71-80$ & 10 & 20 \\
\hline $81-90$ & 3 & 6 \\
\hline \multicolumn{3}{|c|}{ Table 1 } \\
\hline
\end{tabular}

Maximum numbers of cases were in the $7^{\text {th }}$ decade of life followed by $6^{\text {th }}$ decade.

Clinical Presentations- The clinical presentations were as per table below.

\begin{tabular}{|c|c|c|}
\hline Symptoms & No. of Cases & Percentage (\%) \\
\hline Pain abdomen & 25 & 50 \\
\hline Vomiting & 29 & 58 \\
\hline Dyspepsia & 45 & 90 \\
\hline Lump abdomen & 20 & 40 \\
\hline Loss of appetite & 40 & 80 \\
\hline Weight loss & 33 & 66 \\
\hline \multicolumn{2}{|c|}{ Table 2 } \\
\hline
\end{tabular}

In our study dyspepsia was complained by $90 \%$ of patients, loss of appetite in $80 \%$ and weight loss was found in $66 \%$ of patients.

\section{Patterns of Tumours}

The patterns of tumours were as per table below.

\begin{tabular}{|c|c|}
\hline Patterns of Tumour & No. of Cases \\
\hline Intraluminal polypoidal mass & $11(22 \%)$ \\
\hline Focal wall thickening & $30(60 \%)$ \\
\hline Diffuse wall thickening & $9(18 \%)$ \\
\hline \multicolumn{2}{|c|}{ Table 3 } \\
\hline
\end{tabular}

In our study, focal wall thickening was found in maximum no. of cases $(60 \%)$.

\section{Part of Stomach Involved}

In our study, the most common site of involvement was antropyloric region (36\%) followed by fundus and cardia (26\%).

\begin{tabular}{|c|c|c|}
\hline & No. of Cases & Percentage \\
\hline Antropyloric region & 18 & 36 \\
\hline Cardia and fundus & 13 & 26 \\
\hline Body & 10 & 20 \\
\hline Diffuse involvement & 9 & 18 \\
\hline \multicolumn{3}{|c|}{ Table 4 } \\
\hline
\end{tabular}


Involvement of Adjacent Organs

Out of 50 cases of carcinoma stomach in our study, the involvement of adjacent organs was detected by USG and CT as follows.

\begin{tabular}{|c|c|c|}
\hline Adjacent Organs & No. of Cases Detected & \\
\hline & USG & CT \\
\hline Transverse colon & 3 & 6 \\
\hline Liver & 3 & 5 \\
\hline Pancreas & 2 & 4 \\
\hline \multicolumn{2}{|c|}{ Table 5 } \\
\hline
\end{tabular}

Hence, in our study the most common adjacent organ involved by carcinoma stomach were transverse colon $(12 \%)$ followed by liver $(10 \%)$. The involvement of adjacent structures was understaged by USG as compared to CT scan.

Lymph Node Involvement

Out of 50 cases of stomach carcinoma, lymph node involvement were detected by CT in $40(80 \%)$ cases and USG in $34(68 \%)$ cases.

\begin{tabular}{|c|c|c|}
\hline Lymph Node Groups & No. of Cases Detected & \\
\hline & USG & CT \\
\hline Celiac & 23 & 32 \\
\hline Left gastric & 11 & 28 \\
\hline Right gastric & 11 & 15 \\
\hline Gastrohepatic & 16 & 30 \\
\hline Pancreaticosplenic & 10 & 19 \\
\hline Right gastroepiploic & 9 & 14 \\
\hline \multicolumn{2}{|c}{ Table 6 } \\
\hline
\end{tabular}

The enlarged lymph nodes were seen as iso- to hypoattenuated, round-to-oval soft tissue densities with homogeneous/ heterogeneous enhancement on post contrast study. In our study, the most common group of lymph node involved were celiac followed by gastrohepatic. The largest node detected by CT in our study was $30 \mathrm{~mm}$ sized celiac node.

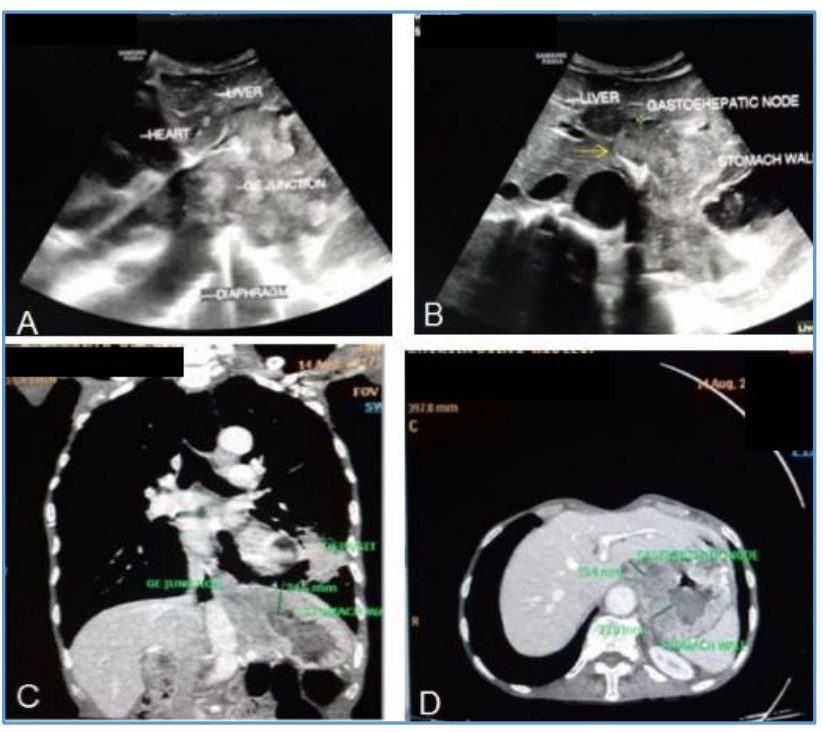

Figure 1. (A and B) USG Image showing Wall Thickening of Fundus and Proximal Body of Stomach extending to GE Junction along with Enlarged Gastrohepatic Node (Yellow Arrow in B), (C) Coronal and Axial CECT Scans showing Heterogeneously enhancing Irregular Wall Thickening of Fundus of Stomach with extension into the GE Junction. Lung deposit is noted in the Left Lower Lobe in Image C along with Enlarged Gastrohepatic Node (19.4 mm GSA Dimension) in D
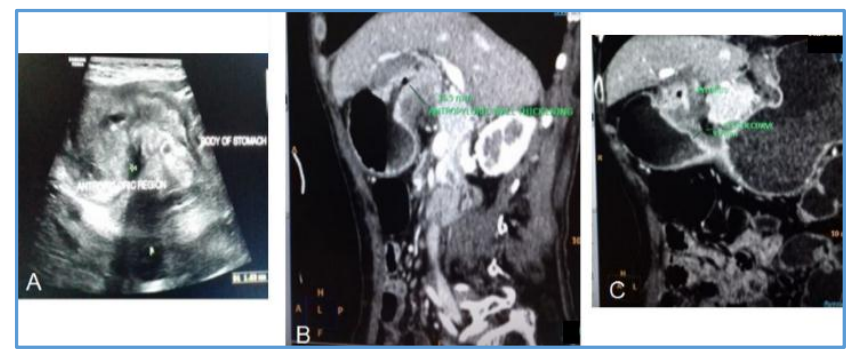

Figure 2. (A) Antropyloric Wall Thickening on USG in a patient presenting with symptoms of Gastric Outlet Obstruction. (B \& C) Same Patient on Sagittal and Coronal CT showing Circumferential Wall Thickening in the Antropyloric region causing Luminal Narrowing and Upstream Distension of the Stomach

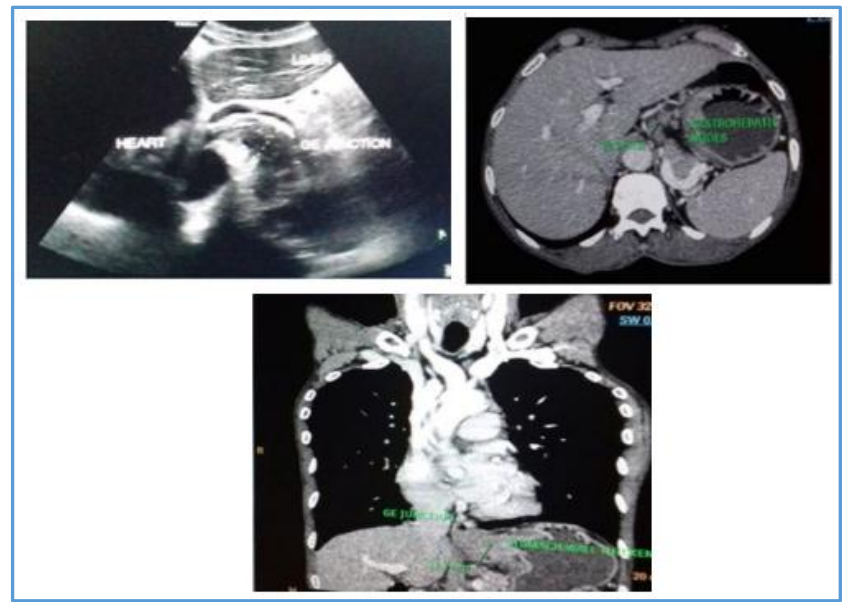

Figure 3. (A) USG Image showing Wall Thickening of the Fundus of the Stomach extending to GE Junction. ( $B$ and $C$ ) CECT Axial and Coronal Images reveals Heterogeneously Enhancing Fundal Wall Thickening extending to the GE Junction. Axial CT Image in addition shows Few Prominent to Enlarged Gastrohepatic Nodes
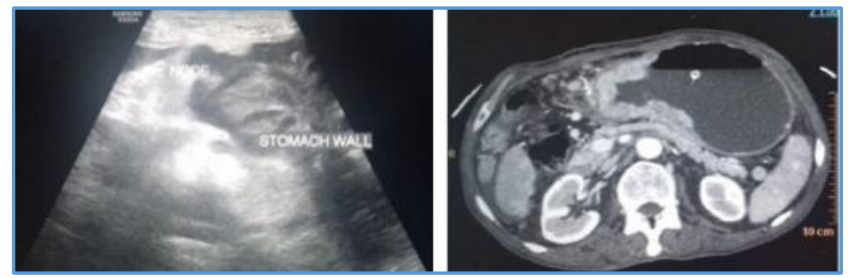

Figure 4. (A) USG showing Circumferential Antropyloric Stomach Wall Thickening. (B) Axial CECT of the same patient showing Transmurally Enhancing Circumferential Antropyloric Wall Thickening extending to greater and lesser curvature with Adjacent Perigastric Nodes and Omental Fat Stranding with Nodularity

\section{DISCUSSION}

In this descriptive study, the number of male patients was 33 (66\%) and female patients was 17 (34\%). Most other studies show a male preponderance in almost all countries with rates of two to four times higher among males than females. $(4,5)$ Age of youngest patient was 37 years and the oldest patient was 83 years. Stomach cancer incidence is known to increase with age with the peak incidence occurring at 60 - 80 years.(6) In our study, maximum number of cases were in the $7^{\text {th }}$ decade of life followed by $6^{\text {th }}$ decade. 
In our study, dyspepsia was complained by $90 \%$ of patients, loss of appetite in $80 \%$ and weight loss was found in $66 \%$ of patients.

Three types of tumour growth was noted- diffuse and focal wall thickening and intraluminal polypoidal mass. Focal wall thickening involving either the proximal (Cardia and Fundus) or distal (Antropyloric Region) stomach was the most common form of stomach carcinoma and represents $60 \%$ of all tumours. Intraluminal polypoidal mass represented $22 \%$ of cases, while the least common form of stomach carcinoma was diffuse pattern, representing $18 \%$ of tumours.

Focal wall thickening of proximal stomach was difficult to diagnose by ultrasonography alone; however, with a high index of clinical suspicion and special emphasis on the GE junction most of the cases were picked up and later confirmed with CT scan of abdomen and HP correlation.

In our study, antropyloric region (36\%) was the most common site of cancer stomach followed by cardia and fundus $(26 \%)$ and body (20\%) of stomach. According to Thomas et al, occurrence of gastric cancer in the stomach is relatively evenly distributed with $30 \%$ occurring in the antrum, $30 \%$ in the body and $40 \%$ in the fundus and cardia.

The most common adjacent organ involved by carcinoma stomach were transverse colon followed by liver and pancreas. The involvement of all these organs was understaged by USG compared to CT scan.

At CT positive lymph nodes are identified on the basis of size, shape and enhancement pattern (i.e. more than 8 - 10 $\mathrm{mm}$ along the short axis, nearly round shape, central necrosis and marked or heterogeneous enhancement). Based on these criteria, the most common group of lymph node involved in our study were celiac followed by gastrohepatic and left gastric stations. CT is better than ultrasound for detecting local spread of the disease and detecting lymphadenopathy. The diagnostic accuracy of CT scan in staging of the disease has been reported in various studies.

In our study out of 13 cases of carcinoma of the cardia and fundus of stomach, longitudinal spread to the distal oesophagus was present in 8 cases (61\%). According to Haren et al oesophageal involvement in carcinoma of the cardia and fundus was $60 \%$, which correlated well with our study.(7)

CT scanning has several pitfalls. CT depicts $75 \%$ of nodes larger than $5 \mathrm{~mm}$ in diameter, but it does not depict tumour in normal-sized nodes. CT is not useful in distinguishing between enlarged nodes due to reactive changes and those due to tumour. Small nodes may contain tumour and large nodes may result from inflammatory causes. Moreover, loss of fat plane between the gastric wall and the left lobe of the liver and between the tumour and pancreas may be due to an inflammatory reaction. In cachectic patients, a loss of fat planes may simulate direct organ invasion.

\section{CONCLUSION}

From our present study of 50 cases of stomach carcinoma, the following conclusions can be drawn: Maximum number of cases was seen occurring between the $5^{\text {th }}$ and $7^{\text {th }}$ decades. Female: male ratio was 1.9: 1. Major risk factor was $\mathrm{H}$. pylori infection; most common pattern of tumour growth was focal wall thickening of stomach and adenocarcinoma was the most common histological type.

Adequate history and strong clinical suspicion is required for diagnosis of Ca Stomach. USG being readily available can be used as a primary tool for detecting cases of carcinoma stomach during routine abdominal sonograms. However, Computed Tomography was found to be superior to ultrasonography in diagnosing and characterising the primary tumour as well as detecting adjacent organ and lymph node involvement.

\section{REFERENCES}

[1] Hallinan JT, Venkatesh SK. Gastric carcinoma: imaging diagnosis, staging and assessment of treatment response. Cancer Imaging 2013;13(2):212-27.

[2] Yoshikawa K, Maruyama K. Characteristics of gastric cancer invading to the proper muscle layer--with special reference to mortality and cause of death. Jpn J Clin Oncol 1985;15(3):499-503.

[3] Ba-Ssalamah A, Prokop M, Uffmann M, et al. Dedicated multidetector CT of the stomach: spectrum of diseases. Radio Graphics 2003;23(3):625-44.

[4] Jemal A, Bray F, Center MM, et al. Global cancer statistics. CA Cancer J Clin 2011;61(2):69-90.

[5] Yeole BB. Trends in cancer incidence in esophagus, stomach, colon, rectum and liver in males in India. Asian Pac J Cancer Prev 2008;9(1):97-100.

[6] Nagini S. Carcinoma of the stomach: a review of epidemiology, pathogenesis, molecular genetics and chemoprevention. World J Gastrointest Oncol 2012;4(7):156-69.

[7] Varia H. Gastric carcinoma imaging. Eugene CL. Chief edr. Updated: Nov 13, 2015. 